Further Section

Appl Neurophysiol 1976;39:327-328

\title{
Subject Index Vol. 39, 1976/77
}

Amygdaloid kindling 302 - nucleus 302 Anesthesics 11 Animal 11 Aphasia 171 Athetosis 251

Back pain 87 Basal ganglia 296 Bilateral thalamotomy 257-Biochemistry 306 Blood sugar 43 Brainstem 296 
- potential 11

- response mappings 34 Excitationofpallidalcells 296 Experimental model 306

Facet denervation 80 Facialpain 122 Focal brain lesions 321 Freely

behaving 11 Frontal opercular cortex 165

Globus pallidus 272 Guidelines 69

Carbamylcholine 1

Cat 239, 302

Cerebellar signs 315

Cerebellothalamocortical projection 239

Cerebrovascular disease 212

Cholinesterase 306

Clinical observations 162

Clinicopathological study 178

Coagulating 87

Computerized axial tomography 212

- transverse axial tomography (CT scanner)

202 Computer program 34,139 Cordotomy 97 Cortical evoked potentials 27

Corticothalamic projection $165 \mathrm{CNV} 261$ 
Heatlosses 69 Hemiballismus 202 High frequency 302 Hippocampus 1 Histochemistry 306 Histogram 50 Human brain stem 139

- pulvinar 158

- thalamus 285

Image matrix 202

Impedance 216

Inhibitory caudatopallidal projection 296

- pallidothalamic projection 296

Instrumentation 77

Intractable pain 114,162

Involuntary disorder 216

Depth stimulation 158 Development 251 Dopamine 306 Drug Treatment 50

Electrical stimulation 139,162 Electrodes 77,80 Electrosurgical 77 Emotional change 261 Evoked cortical potential 158 


\section{Kinesthetic afiferent 285}

Lateral spinothalamic tract 97 i-Dopa 306

L-Dopa-induced dyskinesia 257 Lemniscal, spinoquintothalamic, auditory,

vesti bular, visual, motor, ex t ra pyramidal,

pathways 139 Lesion 306 - generation 77 


\section{Subject Index}

Lesion

- size 69 Leukocytosis 43 Low back pain 80

- frequency 302 Lumbarspine 80

Medical device 77 Monkey 165,239 Motor system 222

Needie scope 58 Neuralnoise 212 Norepinephrine 1

Pain 58, 80,154

- relief 202

Parkinsonism 202,216,257,268, 306 Parkinson's disease 315 Percutaneous cervical cordotomy 97

- cordotomy 114

Postbrachial deafferentation 27

Posterior joints 87

Posture 321 Pulvinar 154

Radiofrequency cordotomy 97

- lesions 97,114,122

- $\quad$ making 69

Reactiontime 321

Readiness potential 268

Recording and stimulation 285

RF heat generation 69

- lesion generation 80

Rhythm 1

Rostral mesencephalic reticulotomy 1 RSA 1

Schiff-Sherrington phenomenon 27 Selspot system 50 Septum 1 Serotonin 1,306 Serum electrolytes 43 Sliceheight 202 
Slow potential' 261 Spectral analysis 50 Spinal cord injury 27

- $\quad$ transection 27

Stereotactic pulvinotomy 162

- surgery $34,139,154$

- $\quad$ ofthediencephalon 202

- thalamotomy 199

Stereotaxic 58

Striatum 272

Stroke til 1

Subcortical stimulation 302

Substantia nigra 272

Subthalamotomy 315

Taste area 165 Telemetry 50 Thalamic lesions 251

- pain 171

- syndrome 199,202 Thalamotomy 43,154,261,311 Thalamus

$34,50,162,171,21^{\wedge} 216,222,306$

- degeneration 178

- $\quad$ primary, combined, presenile 178

Third ventricle 34

Tic douloureux 122 Tremor 50,199, 285, 306, 311

- provocation 311 Trigeminal neuralgia 122

- tractotomy 58

Urethane 11

Vascular lesion 171 VA-VL thalamic nüclëus 272 Ventrolateral nucleus 222

VIMnucleus 285

- thalamotomy 315 VL 306

- nucleus 268

- thalamotomy 321 VOP.VIM 311 VPMpc degeneration 165 